\title{
First Analysis of the Association Between CYP3A4/5, ABCB1 Genetic Polymorphisms and Oxcarbazepine Metabolism and Transport in Chinese Epileptic Patients with Oxcarbazepine Monotherapy and Bitherapy
}

\author{
Ping Wang ${ }^{1}$, Tao Yin ${ }^{1}$, Hong-Ying Ma ${ }^{1}$, Dan-Qi Liu ${ }^{1,2}$, Yang-hao Sheng ${ }^{1,2} \&$ Bo-Ting Zhou ${ }^{1,2}$ \\ ${ }^{1}$ Department of Pharmacy, Xiangya Hospital, Central South University, Changsha Hunan, China. ${ }^{2}$ School of Pharmacy, \\ Central South University, Changsha, Hunan, China.
}

Received, March 25, 2015; Revised, June 18, 2015; Accepted, July 16, 2015; Published, July 17, 2015.

\begin{abstract}
Purpose: Oxcarbazepine (OXC) is widely used in anti-epileptic treatment. Cytochrome P450 3A4 (CYP3A4), cytochrome P450 3A5(CYP3A5), and ATP-binding cassette sub-family B member 1 $(A B C B 1)$ are potential genes involved in OXC metabolisms and transport in vivo. This study aims to examine the genetic effects of $C Y P 3 A 4, C Y P 3 A 5$, and $A B C B 1$ on OXC metabolism and transport in Chinese epileptic patients using OXC as monotherapy and bitherapy with lamotrigine (LTG), levetiracetam (LEV), or valproic acid (VPA). Methods: Sixty-six Chinese epileptic patients were recruited from Xiangya Hospital Central South University, of whom 40 patients were receiving OXC monotherapy, 11 patients were placed in the OXC bitherapy group combined with one enzyme-inducing anti-epileptic drugs (LTG or LEV), and 15 patients were placed in the OXC bitherapy group combined with VPA. Oxcarbazepine and its main metabolite 10-hydrocarbazepine (MHD) plasma concentrations were measured using high performance liquid chromatography (HPLC)-UV method. In addition, eight single nucleotide polymorphisms (SNPs) in CYP3A4, CYP3A5, ABCB1 gene were genotyped by polymerase chain reaction-improved multiple ligase detection reaction (PCR-iMLDR). Results: In the OXC+VPA group, $A B C B 1$ rs2032582 and rs2032582-rs10234411-rs1045642 TAG haplotype were associated with MHD and MHD+OXC plasma concentration before permutation test. In OXC monotherapy and OXC+ LTG/LEV groups, no significant association between genetic polymorphisms in $C Y P 3 A 4 / 5, A B C B 1$ gene and $\mathrm{OXC}$ plasma concentration parameters were observed. Conclusion: $C Y P 3 A 4 / 5$ and $A B C B 1$ genetic variants might not take part in the metabolism and transport of MHD and OXC among epileptic patients using OXC monotherapy and bitherapy in combination with LEV, LTG or VPA.
\end{abstract}

This article is open to POST-PUBLICATION REVIEW. Registered readers (see "For Readers") may comment by clicking on ABSTRACT on the issue's contents page.

\section{INTRODUCTION}

Antiepileptic drugs (AEDs) are considered as the most effective tool for controlling epileptic seizures. Oxcarbazepine (OXC) is a second generation antiepileptic drug used mainly to treat partial epilepsy seizures in epileptic children and adults who use it in monotherapy or adjunctive therapy [1-2]. Like carbamazepine (CBZ) and valproic acid (VPA), the therapeutic range of $\mathrm{OXC}$ is narrow (5-30 $\mu \mathrm{g} / \mathrm{mL})$ [3-4], and OXC blood plasma concentration can be useful in optimizing individual therapy.

CYP3A4/5 consist of functional enzymes of CYP3A subfamilies. Genetic variation in the CYP3A4/5 gene is therefore expected to affect the activities of $C Y P 3 \mathrm{~A} 4 / 5$ enzymes involved in the metabolism of most AEDs [5-7]. Previous studies have shown associations between CYP3A4/5 genetic polymorphisms and plasma concentration of AEDs [8-9]. In a Korean study, presence of the CYP3A5*3 allele is associated with dose-adjusted CBZ plasma concentration in epileptic patients [8]. In African-American patients, the $C Y P 3 A 4 * 1 B$ allele is associated with the pharmacokinetics of CBZ [9]. OXC is oxidized by cytosolic aryl ketone reductase (not a CYP3A4/5 enzyme) in the liver. However, OXC can induce CYP3A4 activity [10], and thus the potential influence of $C Y P 3 A 4 / 5$ variants on OXC plasma concentration parameters

Corresponding Author: Bo-Ting Zhou, Department of Pharmacy, Xiangya Hospital, Central South University, Changsha, Hunan, China, E-mail: botingzhou0918@126.com 
needs to be explored.

The $A B C B 1$ gene encodes P-glycoprotein (P-gp), which is an extensively studied drug efflux transporter. The $A B C B 1$ gene is polymorphic and expression of P-gp can be influenced by $A B C B 1$ mutations [11-13]. Further, some AEDs are substrates of P-gp; for example Zhang et al. [8] have reported that MHD, OXC and CBZ-E are transported by human P-gp. Related findings were fewer but consistent [14-15]. Knowledge of the influence of $A B C B 1$ genetic polymorphisms on OXC plasma concentration parameters may facilitate personalized therapy for epilepsy.

In clinical practice, OXC is commonly administered with other AEDs, and drug interactions may affect OXC and MHD plasma concentration. Whether genetic polymorphisms affect OXC metabolism and transport or whether the effect of genetic polymorphisms on OXC is stronger than the effect caused by drug interactions is not known. The present study was designed to comprehensively evaluate the effects of genetic polymorphisms in $C Y P 3 A 4 / 5$ and $A B C B 1$ genes on OXC plasma concentration parameters in both monotherapy and bitherapy groups of epilepsy patients.

\section{METHODS}

\section{Patients}

Sixty-six epileptic patients treated at Xiangya Hospital in Changsha China were enrolled in the study. The inclusion criteria were: patients were treated with OXC monotherapy or OXC combined with one of VPA, LTG or LEV drugs for more than one month at a consistent dose; diagnosis of partial or generalized seizures according to the International League Against Epilepsy; availability of reliable information on demographics, clinical status, and details of all medication, including doses, time of administration, and duration of therapy. The exclusion criteria were: presence of liver or renal disease; receiving more than two AEDs, any other drugs known to disrupt the activity of drug metabolizing enzymes (including CYP450), such as erycin and cordilox, or any substance with the potential to affect drug metabolizing enzymes, such as traditional Chinese medicine (TCM); poor compliance, including missing doses, adjusting dosage or administrating with the morning dose. Informed consent was obtained from all participants and the study complied with the guidelines of the Medical Ethical
Committee of Xiangya Hospital.

\section{Blood Sampling and Drug Assays}

Blood samples were obtained between 7 am and 9 am before administration of the morning dose to ensure that MHD plasma concentrations were in a steady valley state. Venous blood samples $(5 \mathrm{ml})$ were obtained using an EDTA tube and centrifuged at $3000 \mathrm{rpm}$ for 5 minutes. OXC and MHD plasma concentrations were determined by high performance liquid chromatography (HPLC) (SHIMADZU Inc., Japan). The analytical range for MHD and OXC were $0.234-38.044 \mu \mathrm{g} / \mathrm{mL}$ and 0.239-38.933 $\mu \mathrm{g} / \mathrm{mL}$, respectively. The method had excellent linear correlation $(r=0.999)$ and specificity (intra-and inter-day precision ranged from $0.58-16.67 \%$ and $0.59-14.81 \%$ for MHD and $\mathrm{OXC}$, respectively). OXC+MHD concentration represented the sum of OXC and MHD.

\section{Genotyping}

Genomic DNA was extracted from whole blood using the method of phenol-chloroform. We selected eight SNPs including CYP3A4 rs4646440, rs2242480, CYP3A5 rs15524, rs776746, $A B C B 1$ rs1045642, rs2032582, rs10234411, rs1128503 for the current project. All were potentially functional SNPs, with minor allele frequencies (MAF) of more than $10 \%$ in the Hapmap HCB (Han Chinese in Beijing). SNP positions were obtained from http://hapmap.ncbi.nlm.nih.gov. Genetic polymorphisms were detected using polymerase chain reaction-improved multiple ligase detection reaction (PCR- iMLDR) according to the manufacture's instructions (Center for Genetic \& Genomic Analysis, Genesky Biotechnologies Inc., Shanghai). All eight SNPs were successfully genotyped in 66 patients with call rates of $100 \%$.

\section{STATISTICAL ANALYSIS}

Statistical comparisons of basic characteristics among OXC monotherapy and bitherapy groups were done using an analysis of variance (ANOVA) or a $\chi^{2}$ test. Pearson (measurement data) and Spearman (enumeration data) correlation tests were conducted to detect potential clinical factors affecting OXC plasma concentration parameters, and the significant clinical factors were used to adjust concentration parameters. These analyses were performed in SPSS (version 16.0, SPSS Inc., IL, USA). Haploview 4.2 was used to generate genetic haplotypes. PLINK (version 1.0.7) [16] was 
used to perform Hardy-Weinberg equilibrium calculations, to analyze the association between SNPs and adjusted OXC plasma concentration parameters, and for haplotype analysis. Permutation tests $(n=10000)$ were used to assess significance in multiple testing of genotype-phenotype correlations. A two-tailed $\mathrm{p}$ of less than 0.05 was considered to be statistically significant.

\section{RESULTS}

Of the enrolled epileptic patients, 40 patients received OXC monotherapy and 26 received bitherapy with another AED: LTG (6), LEV (5), or VPA (15). The OXC+LTG and OXC+LEV groups were combined for adequate sample size. The clinical characteristics of the 66 epileptic patients are summarized in Table 1; the three treatment groups did not differ for any of these variables.

MHD and MHD+OXC plasma concentrations were positively correlated with $\mathrm{OXC}$ dose requirements $(\mathrm{r}=0.624, \mathrm{p}<0.001$ and $\mathrm{r}=0.628, \mathrm{p}$ $<0.001)$. However, OXC plasma concentration, while related to age $(r=0.503, p<0.001)$, was not significantly correlated with OXC dose $(\mathrm{r}=0.248$, $\mathrm{p}=0.097$; Figure 1). Dose-adjusted MHD, age-adjusted OXC, and dose-adjusted MHD+OXC plasma concentrations were tested for treatment (OXC monotherapy and bitherapy) group effects by ANOVA; treatment groups differences were not statistically significant (Figure 2).

The minor allele frequency (MAF) of SNPs used ranged from $15.9 \%$ to $40.6 \%$ (mean $=30.9 \%$ ) and no SNPs were significantly out of Hardy-Weinberg equilibrium (Table 2). In the OXC monotherapy group, no significant associations of SNPs with OXC plasma concentration parameters were present. In the OXC +VPA biotherapy group, $A B C B 1$ SNP rs2032582 was associated with MHD $(\mathrm{p}=0.017)$ and MHD+OXC $(\mathrm{p}=0.019)$ plasma concentration, but the associations were not significant after correcting for multiple tests (Table 3).

Haplotype analysis of SNPs in Haploview suggested SNPs fell into three blocks according to linkage disequilibrium levels (Figure 3). As with SNPs, we analyzed the association of adjusted OXC plasma concentration parameters with $C Y P 3 A 4 / 5$ and $A B C B 1$ haplotypes. Consistent with the SNPs association analysis, in the OXC+VPA group, $A B C B 1 \quad$ SNPs group, rs2032582-rs10234411-rs1045642 TAG haplotype is significantly associated with MHD $(\mathrm{p}=0.017)$ and MHD+OXC ( $\mathrm{p}=0.019)$ plasma concentration, using p-values uncorrected for multiple tests (Table $4)$.

\section{DISCUSSION}

To the best of our knowledge, this is the first study to explore the effects of genetic polymorphisms on OXC plasma concentration parameters. Using genetic association analysis, we demonstrate that $A B C B 1$ SNP rs2032582, and the rs2032582-rs10234411-rs1045642 TAG haplotype are associated with MHD and MHD+OXC plasma concentration in OXC+VPA group before permutation test; in OXC monotherapy and OXC+ LTG/LEV group. Our study did not show a connection between $C Y P 3 A 4 / 5, A B C B 1$ genetic variants and MHD, MHD+OXC plasma concentrations.

$A B C B 1$ rs2032582, rs10234411, rs1045642, rs1128503 SNPs are extensively studied SNPs, hence it is possible that the sequence change may influence the transcription of P-gp. Notably, SNPs rs2032582 was the first SNP discovered from P-gp of human [17]. The association of rs 2032582 with carbamazepine resistance in monotherapy has been reported among Malays [17]. Similarly, Seo et al. [18] demonstrated that $A B C B 1$ rs2032582 may influence carbamazepine responsiveness in monotherapy or polytherapy among Japanese, but did not report on the association of $A B C B 1$ genetic polymorphisms and $\mathrm{OXC}$ plasma concentration parameters. Our study on a Chinese population reveals that SNPs rs2032582 is significantly associated with MHD, MHD+OXC plasma concentration in OXC+VPA group. Although associations were not statistically significant after a permutation test to correct for multiple tests, it provides genetic evidence for further study of OXC metabolism and transport.

CYP3A4 rs4646440, rs 2242480 and CYP3A5 rs776746, rs 15524 probably contribute to the variability of enzymatic activity [19-20]. In our association study, we found CYP3A4/5 SNPs and haplotypes have no association with OXC plasma concentration parameters. Although it has been reported that $\mathrm{OXC}$ is capable of inducing the metabolism of other drugs via CYP3A4, and the inductive effect is clinically significant [21]. Our results suggest that $\mathrm{OXC}$ is only partially affected by $C Y P 3 A 4 / 5$ activity, and it has the potential to replace CBZ, especially for $C Y P 3 A 5 * 3$ epileptic patients. 
Combining the SNPs association analysis, we found that $A B C B 1$ rs2032582-rs10234411-rs1045642 TAG was associated with MHD, MHD+OXC plasma concentration before permutation test in OXC+VPA group. The result was consistent with the SNPs association analysis. As only rs2032582 was found in SNPs association analysis, rs10234411 and rs1045642 showed no associations, it indicates that SNPs in linkage disequilibrium maybe stronger markers for association analysis.

In our routine therapeutic drug monitoring, we monitored more and more OXC plasma concentration of epileptic patients and observed that the variability among individuals was large. As the first study to investigate the effect of $C Y P 3 A 4 / 5$, $A B C B 1$ genetic polymorphism on OXC metabolism and transport, our findings reveal the importance of evaluating the role of genetic variation on inter-individual variation of OXC plasma concentration, especially when patients are in good compliance. On the basis of our results, the future studies can replicate our research and explore the regulatory mechanism of $A B C B 1$ genetic variations. However, there are some limitations of this study. Drug-drug interactions might affect OXC and MHD plasma concentration, and this effect is confounded in the association analysis between genetic polymorphisms and OXC metabolism.
Nevertheless, MHD and OXC plasma concentration have no significant differences among OXC monotherapy, OXC+VPA group and $\mathrm{OXC}+$ LTG/LEV treatment groups; thus any effect of this confounding factor on our results is not significant. Our sample size was not large, and it may be a factor that affecting the results after permutation test.

In summary, the present study investigated the association of $C Y P 3 A 4 / 5, \quad A B C B 1$ genetic polymorphisms with $\mathrm{OXC}$ plasma concentration parameters, and found no significant connection after correcting for multiple tests. Therefore, the results suggested that $C Y P 3 A 4 / 5, A B C B 1$ genetic variations do not influence OXC metabolism and transport in epileptic patients using OXC monotherapy or bitherapy combined with LTG, LEV or VPA. To confirm the findings, replication of the study in other cohorts is required.

\section{ACKNOWLEDGEMENTS}

We thank all study participants. This work was supported by the National Natural Science Foundation of China (81373493) and the Hunan Provincial Science Foundation of China (12JJ6083).

Table1. Summary data (mean \pm SD) for epileptic patients in each of the three treatment group (total $n=66$ ).

\begin{tabular}{|c|c|c|c|c|}
\hline Characteristics & $\begin{array}{l}\text { OXC monotherapy } \\
(\mathrm{n}=40)\end{array}$ & $\begin{array}{c}\text { OXC+VPA } \\
(n=15)\end{array}$ & $\begin{array}{l}\text { OXC+LTG/LEV } \\
(\mathrm{n}=11)\end{array}$ & $\mathrm{P}$ \\
\hline Gender (M/F) & $22 / 18$ & $10 / 5$ & $7 / 4$ & $0.695^{\mathrm{a}}$ \\
\hline Age(years) & $16.35 \pm 14.73$ & $14.97 \pm 13.25$ & $11.70 \pm 12.97$ & $0.629^{b}$ \\
\hline Weight(kg) & $38.83 \pm 20.18$ & $42.35 \pm 26.01$ & $31.68 \pm 22.09$ & $0.470^{b}$ \\
\hline Height (m) & $1.35 \pm 0.35$ & $1.61 \pm 0.18$ & $1.56 \pm 0.24$ & $0.166^{b}$ \\
\hline $\mathrm{BMI}\left(\mathrm{kg} / \mathrm{m}^{2}\right)$ & $19.19 \pm 3.82$ & $21.93 \pm 4.50$ & $20.72 \pm 4.26$ & $0.287^{b}$ \\
\hline $\operatorname{Dose}(\mathrm{g})$ & $0.57 \pm 0.42$ & $0.61 \pm 0.55$ & $0.60 \pm 0.32$ & $0.921^{\mathrm{b}}$ \\
\hline MHD $(\mu \mathrm{g} / \mathrm{mL})$ & $11.44 \pm 7.61$ & $9.54 \pm 4.30$ & $11.24 \pm 5.72$ & $0.643^{b}$ \\
\hline $\mathrm{OXCBZ}(\mu \mathrm{g} / \mathrm{mL})$ & $0.39 \pm 0.50$ & $0.21 \pm 0.13$ & $0.74 \pm 0.81$ & $0.113^{b}$ \\
\hline $\begin{array}{l}\text { MHD+OXCBZ } \\
(\mu \mathrm{g} / \mathrm{mL})\end{array}$ & $11.72 \pm 7.81$ & $9.65 \pm 4.31$ & $11.85 \pm 6.11$ & $0.590^{\mathrm{b}}$ \\
\hline \multicolumn{5}{|c|}{$\begin{array}{l}\text { OXC: oxcarbazepine; MHD: monohydroxy derivative; VPA: Valproic acid; LTG: Lamotrigine; LEV: } \\
\text { Levetiracetam; M/F: Male/Female; BMI: body mass index; a: p-value was calculated between OXC monotherapy } \\
\text { group and bitherapy groups by } \chi^{2} \text { test; b: p-values were calculated by ANOVA. }\end{array}$} \\
\hline
\end{tabular}


Table 2. Characteristics of the eight SNPs detected $(n=66)$

\begin{tabular}{llllllll}
\hline CHR & Gene & SNP & Locus & Position & Allele & MAF & HWE \\
\hline 7 & $A B C B 1$ & rs1045642 & Exon & 87138645 & A/G & 0.394 & 0.069 \\
7 & $A B C B 1$ & rs2032582 & Exon & 87160618 & G/T/A & 0.159 & 0.194 \\
7 & $A B C B 1$ & rs10234411 & Intron & 87164892 & T/A & 0.409 & 0.062 \\
7 & $A B C B 1$ & rs1128503 & Exon & 87179601 & G/A & 0.318 & 0.569 \\
7 & $C Y P 3 A 5$ & rs15524 & 3'UTR & 99245914 & G/A & 0.371 & 0.607 \\
7 & $C Y P 3 A 5$ & rs776746 & Intron & 99270539 & A/G & 0.348 & 1.000 \\
7 & $C Y P 3 A 4$ & rs4646440 & Intron & 99360870 & A/G & 0.227 & 0.726 \\
7 & CYP3A4 & rs2242480 & Intron & 99361466 & A/G & 0.250 & 1.000 \\
\hline
\end{tabular}

CHR: chromosome; SNP: single-nucleotide polymorphism; MAF: minor allele frequency; HWE: p-value for analysis of Hardy-Weinberg equilibrium

Table 3. Associations of $C Y P 3 A 4 / 5$ and $A B C B 1$ SNPs with adjusted plasma concentrations of OXC, MHD, or $\mathrm{MHD}+\mathrm{OXC}$

\begin{tabular}{|c|c|c|c|c|c|c|c|c|c|}
\hline \multirow[t]{2}{*}{ SNP } & \multicolumn{3}{|l|}{$\mathrm{OXC}$} & \multicolumn{3}{|c|}{$\mathrm{OXC}+\mathrm{VPA}$} & \multicolumn{3}{|c|}{ OXC+LTG/LEV } \\
\hline & $\mathrm{p}_{\mathrm{MHD}}$ & poxc & $\mathrm{p}_{\mathrm{MHD}+\mathrm{OXC}}$ & $\mathrm{p}_{\mathrm{MHD}}$ & poxc & $\mathrm{p}_{\mathrm{MHD}+\mathrm{OXC}}$ & $\mathrm{p}_{\mathrm{MHD}}$ & poxc & $\mathrm{p}_{\mathrm{MHD}+\mathrm{OXC}}$ \\
\hline rs1045642 & 0.781 & 0.059 & 0.916 & 0.345 & 0.505 & 0.372 & 0.366 & 0.389 & 0.420 \\
\hline rs2032582 & 0.959 & 0.673 & 0.931 & 0.017 & 0.561 & 0.019 & 0.097 & 0.309 & 0.087 \\
\hline rs10234411 & 0.789 & 0.070 & 0.919 & 0.317 & 0.457 & 0.345 & 0.360 & 0.340 & 0.419 \\
\hline rs1128503 & 0.280 & 0.037 & 0.221 & 0.697 & 0.111 & 0.752 & 0.148 & 0.916 & 0.157 \\
\hline rs15524 & 0.546 & 0.401 & 0.511 & 0.257 & 0.652 & 0.278 & 0.743 & 0.829 & 0.763 \\
\hline rs776746 & 0.540 & 0.530 & 0.518 & 0.450 & 0.460 & 0.480 & 0.682 & 0.827 & 0.704 \\
\hline rs4646440 & 0.403 & 0.313 & 0.370 & 0.117 & 0.461 & 0.135 & 0.903 & 0.585 & 0.942 \\
\hline rs2242480 & 0.429 & 0.413 & 0.404 & 0.117 & 0.461 & 0.135 & 0.560 & 0.891 & 0.579 \\
\hline
\end{tabular}

OXC: oxcarbazepine; MHD: monohydroxy derivative; VPA: Valproic acid; LTG :Lamotrigine; LEV: Levetiracetam; Numbers in the table are p-values for tests of association obtained from PLINK; Bold p values indicate significant associations between SNPs and adjusted concentrations before permutation test.
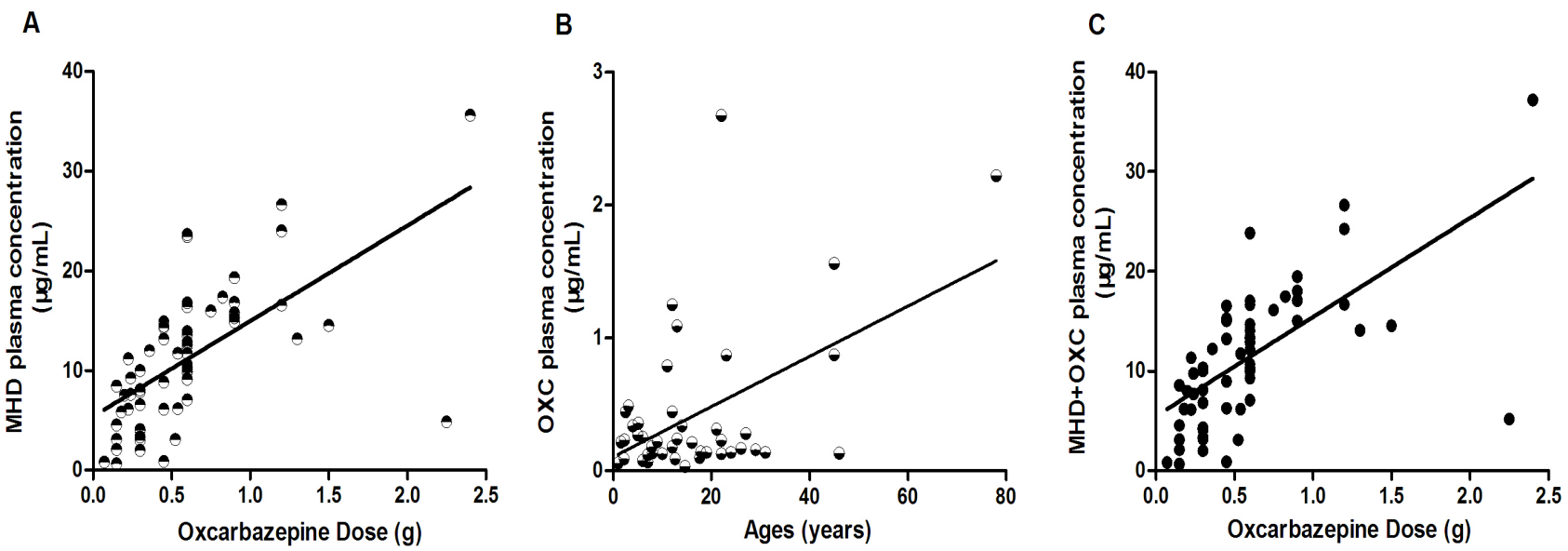

Figure 1. Relationship between oxcarbazepine (OXC) dose and MHD plasma concentration (A) and relationship between ages and OXC plasma concentration (B) and relationship between oxcarbazepine (OXC) dose and MHD + OXC plasma concentration (C). 

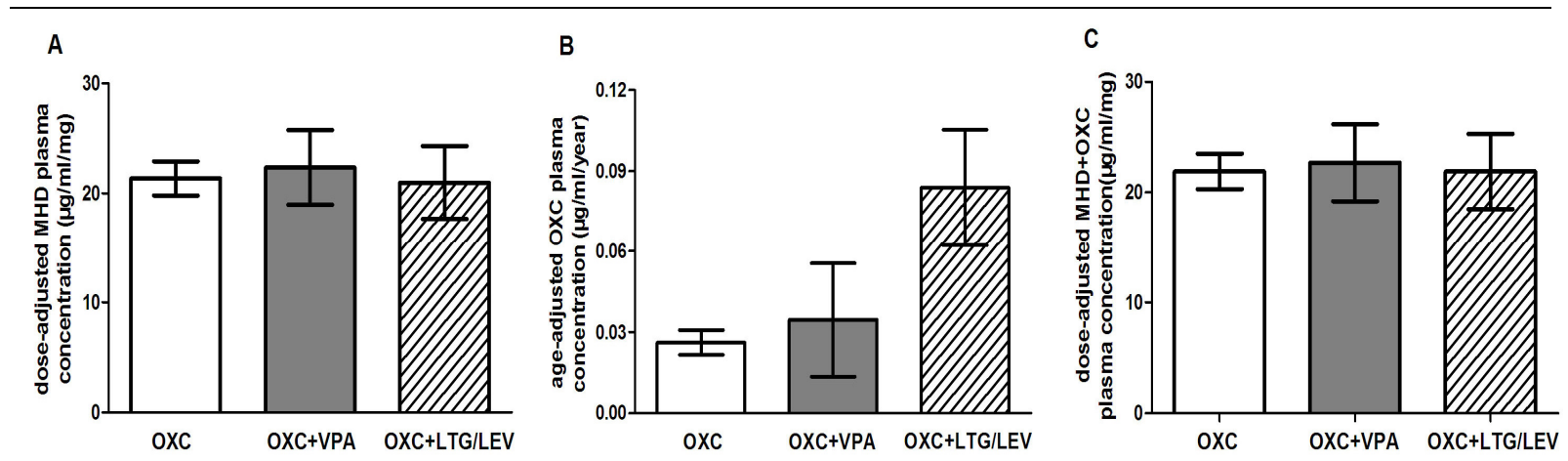

Figure 2. Comparison of OXC plasma concentration parameters by treatment groups: OXC monotherapy, bitherapy with VPA, and bitherapy with LTG/LEV. A: dose-adjusted MHD plasma concentration B: age-adjusted OXC plasma concentration; C: dose-adjusted MHD+OXC plasma concentration. None of the treatments differed by ANOVA.

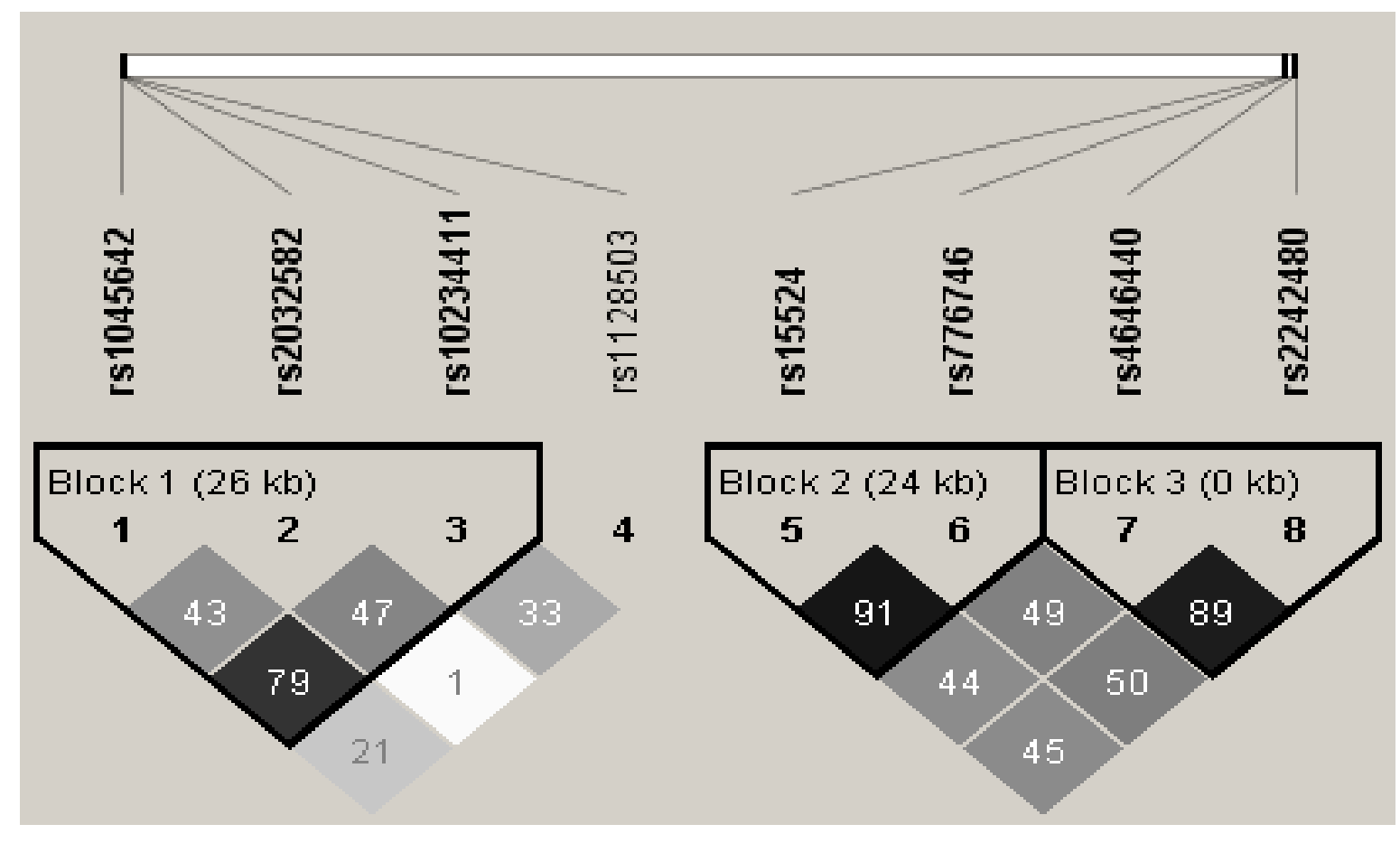

Figure 3. The linkage disequilibrium of 8 single nucleotide polymorphisms (SNPs) in $C Y P 3 A 4 / 5, A B C B 1$ in all patients. SNPs 1-4 are in $A B C B 1$, SNPs 5-6 are in $C Y P 3 A 5$, and SNPs 7-8 are in $C Y P 3 A 4$; Haploview analysis of linkage disequilibrium for all patients between pairs of SNPs are shown in diamonds, with darker diamonds for greater R-squared; R-squared values multiplied by 100 are shown in diamonds; Bold numbers represent SNPs of strong linkage disequilibrium.

\section{REFERENCES}

1. Northam, R S, Hernandez, A W, Litzinger, M J, Minecan, D N, Glauser, T A, Mangat, S, Zheng, C, Souppart, C, Sturm, Y. Oxcarbazepine in infants and young children with partial seizures. Pediatr Neurol, 2005; 33: 337-344

2. Smith, $\mathrm{P}$ E. Clinical recommendations for oxcarbazepine. Seizure, 2001; 10: 87-91

3. Breton, H, Cociglio, M, Bressolle, F, Peyriere, H,
Blayac, J P, Hillaire-Buys, D. Liquid chromatography-electrospray mass spectrometry determination of carbamazepine, oxcarbazepine and eight of their metabolites in human plasma. J Chromatogr B Analyt Technol Biomed Life Sci, 2005; 828: 80-90

4. Neels, H M, Sierens, A C, Naelaerts, K, Scharpe, S L, Hatfield, G M, Lambert, W E. Therapeutic drug monitoring of old and newer anti-epileptic drugs. Clin Chem Lab Med, 2004; 42: 1228-1255 
5. Huang, W, Lin, Y S, Mcconn, D N, Calamia, J C, Totah, R A, Isoherranen, N, Glodowski, M, Thummel, K E. Evidence of significant contribution from $C Y P 3 A 5$ to hepatic drug metabolism. Drug Metab Dispos, 2004; 32: 1434-1445

6. Jaramillo, N M, Galindo, I F, Vazquez, A O, Cook, H J, Llerena, A, Lopez, M L. Pharmacogenetic potential biomarkers for carbamazepine adverse drug reactions and clinical response. Drug Metabol Drug Interact, 2014; 29: 67-79

7. Lynch, T, Price, A. The effect of cytochrome P450 metabolism on drug response, interactions, and adverse effects. Am Fam Physician, 2007; 76: 391-396

8. Zhang, C, Zuo, Z, Kwan, P, Baum, L. In vitro transport profile of carbamazepine, oxcarbazepine, eslicarbazepine acetate, and their active metabolites by human P-glycoprotein. Epilepsia, 2011; 52: 1894-1904

9. Puranik, Y G, Birnbaum, A K, Marino, S E, Ahmed, G, Cloyd, J C, Remmel, R P, Leppik, I E, Lamba, J $\mathrm{K}$. Association of carbamazepine major metabolism and transport pathway gene polymorphisms and pharmacokinetics in patients with epilepsy. Pharmacogenomics, 2013; 14: 35-45

10. Hogler, W, Wudy, S A, Luef, G, Hartmann, M F, Rauchenzauner, M. Oxcarbazepine accelerates cortisol elimination via cytochrome P450 3A4 induction. Arch Dis Child, 2010; 95: 1065

11. Marzolini, C, Paus, E, Buclin, T, Kim, R B. Polymorphisms in human MDR1 (P-glycoprotein): recent advances and clinical relevance. Clin Pharmacol Ther, 2004; 75: 13-33

12. Ozgon, G O, Bebek, N, Gul, G, Cine, N. Association of MDR1 (C3435T) polymorphism and resistance to carbamazepine in epileptic patients from Turkey. Eur Neurol, 2008; 59: 67-70

13. Zimprich, F, Sunder-Plassmann, R, Stogmann, E, Gleiss, A, Dal-Bianco, A, Zimprich, A, Plumer, S, Baumgartner, C, Mannhalter, C. Association of an ABCB1 gene haplotype with pharmacoresistance in temporal lobe epilepsy. Neurology, 2004; 63: 1087-1089

14. Clinckers, R, Smolders, I, Meurs, A, Ebinger, G, Michotte, Y. Quantitative in vivo microdialysis study on the influence of multidrug transporters on the blood-brain barrier passage of oxcarbazepine: concomitant use of hippocampal monoamines as pharmacodynamic markers for the anticonvulsant activity. J Pharmacol Exp Ther, 2005; 314: 725-731

15. Marchi, N, Guiso, G, Rizzi, M, Pirker, S, Novak, K, Czech, T, Baumgartner, C, Janigro, D, Caccia, S, Vezzani, A. A pilot study on brain-to-plasma partition

10,11-dyhydro-10-hydroxy-5H-dibenzo(b,f)azepine5-carboxamide and MDR1 brain expression in epilepsy patients not responding to oxcarbazepine. Epilepsia, 2005; 46: 1613-1619
16. Purcell, S, Neale, B, Todd-Brown, K, Thomas, L, Ferreira, M A, Bender, D, Maller, J, Sklar, P, de Bakker, P I, Daly, M J, Sham, P C. PLINK: a tool set for whole-genome association and population-based linkage analyses. Am J Hum Genet, 2007; 81: 559-575

17. Subenthiran, S, Abdullah, N R, Joseph, J P, Muniandy, P K, Mok, B T, Kee, C C, Ismail, Z, Mohamed, Z. Linkage disequilibrium between polymorphisms of $\mathrm{ABCB} 1$ and $\mathrm{ABCC} 2$ to predict the treatment outcome of Malaysians with complex partial seizures on treatment with carbamazepine mono-therapy at the Kuala Lumpur Hospital. PLoS One, 2013; 8: e64827

18. Seo, T, Ishitsu, T, Ueda, N, Nakada, N, Yurube, K, Ueda, K, Nakagawa, K. ABCB1 polymorphisms influence the response to antiepileptic drugs in Japanese epilepsy patients. Pharmacogenomics, 2006; 7: 551-561

19. Chen, C H, Wang, S C, Tsou, H H, Ho, I K, Tian, J $\mathrm{N}, \mathrm{Yu}, \mathrm{C}$ J, Hsiao, C F, Chou, S Y, Lin, Y F, Fang, K C, Huang, C L, Su, L W, Fang, Y C, Liu, M L, Lin, K M, Hsu, Y T, Liu, S C, Chen, A, Liu, Y L. Genetic polymorphisms in $C Y P 3 \mathrm{~A} 4$ are associated with withdrawal symptoms and adverse reactions in methadone maintenance patients. Pharmacogenomics, 2011; 12: 1397-1406

20. Kuehl, P, Zhang, J, Lin, Y, Lamba, J, Assem, M, Schuetz, J, Watkins, P B, Daly, A, Wrighton, S A, Hall, S D, Maurel, P, Relling, M, Brimer, C, Yasuda, $\mathrm{K}$, Venkataramanan, R, Strom, S, Thummel, K, Boguski, M S, Schuetz, E. Sequence diversity in $C Y P 3 \mathrm{~A}$ promoters and characterization of the genetic basis of polymorphic CYP3A5 expression. Nat Genet, 2001; 27: 383-391

21. Andreasen, A H, Brosen, K, Damkier, P. A comparative pharmacokinetic study in healthy volunteers of the effect of carbamazepine and oxcarbazepine on cyp3a4. Epilepsia, 2007; 48: 490-496 
J Pharm Pharm Sci (www.cspsCanada.org) 18(2) 258 - 265, 2015

Table 4. Associations between $C Y P 3 A 4 / 5$ or $A B C B 1$ haplotypes and adjusted OXC plasma concentration parameters

\begin{tabular}{|c|c|c|c|c|c|c|c|c|c|c|c|c|c|c|c|}
\hline \multirow[t]{2}{*}{ Block } & \multicolumn{5}{|l|}{$\mathrm{OXC}$} & \multicolumn{5}{|l|}{$\mathrm{OXC}+\mathrm{VPA}$} & \multicolumn{5}{|c|}{ OXC+LTG/LEV } \\
\hline & Haplotype & Freq & $\mathrm{p}_{\mathrm{MHD}}$ & poxc & $\mathrm{p}_{\mathrm{MHD}+\mathrm{OXC}}$ & Haplotype & Freq & $\mathrm{p}_{\mathrm{MHD}}$ & poxc & $\mathrm{p}_{\mathrm{MHD}+\mathrm{OXC}}$ & Haplotype & Freq & $\mathrm{p}_{\mathrm{MHD}}$ & poxc & $\mathrm{p}_{\mathrm{MHD}+\mathrm{OXC}}$ \\
\hline rs 15524 & GT & 0.325 & 0.540 & 0.530 & 0.518 & GT & 0.333 & 0.450 & 0.460 & 0.480 & $\mathrm{AC}$ & 0.464 & 0.743 & 0.829 & 0.763 \\
\hline \multirow{2}{*}{-rs776746 } & GC & 0.013 & 0.952 & 0.343 & 0.980 & GC & 0.033 & 0.207 & 0.600 & 0.209 & GC & 0.036 & 0.927 & 0.965 & 0.926 \\
\hline & $\mathrm{AC}$ & 0.663 & 0.546 & 0.401 & 0.511 & $\mathrm{AC}$ & 0.633 & 0.257 & 0.652 & 0.278 & GT & 0.500 & 0.682 & 0.827 & 0.704 \\
\hline rs4646440 & AT & 0.213 & 0.403 & 0.313 & 0.370 & AT & 0.167 & 0.117 & 0.461 & 0.135 & AT & 0.393 & 0.903 & 0.585 & 0.942 \\
\hline \multirow{2}{*}{-rs2242480 } & GT & 0.025 & 0.907 & 0.599 & 0.873 & - & & & & & GT & 0.036 & 0.364 & 0.357 & 0.336 \\
\hline & GC & 0.763 & 0.429 & 0.413 & 0.404 & GC & 0.833 & 0.117 & 0.461 & 0.135 & GC & 0.571 & 0.560 & 0.891 & 0.579 \\
\hline rs2032582 & ATA & 0.387 & 0.797 & 0.082 & 0.922 & ATA & 0.367 & 0.345 & 0.505 & 0.372 & ATA & 0.321 & 0.366 & 0.389 & 0.420 \\
\hline -rs 10234411 & AAA & 0.019 & 0.890 & 0.619 & 0.928 & - & & & & & - & & & & \\
\hline \multirow[t]{3}{*}{-rs1045642 } & ATG & 0.013 & 0.932 & 0.555 & 0.976 & ATG & 0.067 & 0.828 & 0.809 & 0.840 & ATG & 0.071 & 0.873 & 0.743 & 0.897 \\
\hline & TAG & 0.144 & 0.965 & 0.736 & 0.943 & TAG & 0.200 & 0.017 & 0.561 & 0.019 & TAG & 0.179 & 0.097 & 0.309 & 0.087 \\
\hline & $\mathrm{AAG}$ & 0.431 & 0.807 & 0.040 & 0.953 & AAG & 0.367 & 0.446 & 0.147 & 0.420 & AAG & 0.429 & 0.724 & 0.052 & 0.621 \\
\hline
\end{tabular}

OXC: oxcarbazepine; MHD: monohydroxy derivative; VPA: Valproic acid; LTG: Lamotrigine; LEV: Levetiracetam; -: represent no data. ; Numbers in the table are p-values

for tests of association obtained from PLINK; Bold p-values indicate significant associations between haplotypes and adjusted concentrations before permutation test. 